\title{
Critical interfaces in the random-bond Potts model
}

\author{
Jesper L. Jacobsen, ${ }^{1}$ Pierre Le Doussal, ${ }^{1}$ Marco Picco, ${ }^{2}$ Raoul Santachiara,${ }^{1}$ and Kay Jörg Wiese ${ }^{1}$ \\ ${ }^{1}$ CNRS-Laboratoire de Physique Théorique de l'Ecole Normale Supérieure, 24 rue Lhomond, 75231 Paris, France. \\ ${ }^{2}$ CNRS-LPTHE, Universités Paris 6 et Paris 7, 4 Place Jussieu, 75005 Paris, France
}

\begin{abstract}
We study geometrical properties of interfaces in the random-temperature $q$-states Potts model as an example of a conformal field theory weakly perturbed by quenched disorder. Using conformal perturbation theory in $q-2$ we compute the fractal dimension of Fortuin Kasteleyn domain walls. We also compute it numerically both via the Wolff cluster algorithm for $q=3$ and via transfer-matrix evaluations. We also obtain numerical results for the fractal dimension of spin clusters interfaces for $q=3$. These are found numerically consistent with the duality $\kappa^{\mathrm{spin}} \kappa^{\mathrm{FK}}=16$ as expressed in putative SLE parameters.
\end{abstract}

The discovery of the Schramm Löwner Evolution (SLE) has strongly revived interest in geometrical properties of interfaces in 2-dimensional statistical physics. SLE provides a rigorous classification, with a single parameter $\kappa$, of probability measures on non-crossing random fractal curves, which satisfy both conformal invariance and the domain Markov property [1]. Interfaces and similar geometric objects defined in pure 2-dimensional critical models are conjectured, and in some cases proven, to satisfy both requirements in the continuum limit. SLE hence describes such diverse systems as percolation $\kappa=6$, self-avoiding walks $\kappa=4 / 3$, loop-erased random walks $\kappa=2$ and level lines of height models $\kappa=4$ [2]. It applies to the Ising and 3-states Potts interfaces, both for spin clusters $(\kappa=3$ and $\kappa=10 / 3$, respectively) and the dual Fortuin Kasteleyn (FK) clusters $(\kappa=16 / 3$ and $\kappa=24 / 5)$, with a duality $\kappa \leftrightarrow \kappa^{\prime}=16 / \kappa$. While these models have been described, prior to SLE, using conformal field theory (CFT), SLE bridges the gap between the algebraic approach of CFT and the geometry of interfaces. The present connections between SLE and CFT 2 focus on boundary-condition changing operators, which generate the curves. They give $d_{\mathrm{f}}=1+\kappa / 8$ for the fractal dimension of the interface, i.e. the hull of the SLE trace. Extensions beyond non-minimal CFT [3] are rare.

A tantalizing question is whether CFT methods and SLE help to understand a broader class of scale-invariant $2 \mathrm{~d}$ complex systems, such as systems with quenched disorder or far from equilibrium. Numerical studies indicate that zero-vorticity lines in 2D-turbulence [4] and domain walls in spin-glasses [5] may be described by SLE. These examples are "far" from any pure CFT, thus the situation may be more favorable for models which are "weak perturbations" of a known CFT. This is e.g. the case for the $q$-states Potts model, perturbed by quenched random bond (i.e., temperature) disorder, known to exhibit a stable weak-disorder fixed point for $q>2$, perturbatively accessible in a $q-2$ expansion. This has been studied using conformal perturbation theory [6, 7] and transfer-matrix methods [8]. However, geometric properties of interfaces which are crucial for future comparison to SLE were to our knowledge not investigated.
The aim of this Letter is to present results for the fractal dimension of domain walls in the random-temperature Potts model. These are obtained by analytical calculation using conformal perturbation theory inspired by [6, 7], and from two types of large-scale numerics: Monte Carlo simulations using the efficient Wolf-algorithm [10, which allow to keep track of both spin and FK clusters in the same simulation, and transfer-matrix calculations, whose advantage is to make close contact with CFT. The results of all three methods agree nicely.

Let us recall the definition of the model: In terms of the spin variables $\sigma_{i}=\{1, \cdots, q\}$ at lattice site $i$, the partition function of the $q$-states Potts model is

$$
\mathcal{Z}=\sum_{\left\{\sigma_{i}\right\}} \mathrm{e}^{\beta \sum_{\langle i j\rangle} J_{i j} \delta_{\sigma_{i} \sigma_{j}}} \sim \sum_{\left\{\sigma_{i}\right\}} \prod_{\langle i j\rangle}\left[1-\mathrm{p}_{i j}+\mathrm{p}_{i j} \delta_{\sigma_{i} \sigma_{j}}\right]
$$

where the sum runs over nearest-neighbor bonds $\langle i, j\rangle$. The last expression is the spin-cluster expansion, noting $1-\mathrm{p}_{i j}=\mathrm{e}^{-\beta J_{i j}}$. By expanding in $\mathrm{p}_{i j}$, it can be rewritten in terms of the Fortuin-Kasteleyn (FK) clusters, composed by placing a bond between neighboring sites with probability $\mathrm{p}_{i j}$. The pure ferromagnetic model has $J_{i j}=J>0, \mathrm{p}_{i j}=\mathrm{p}$, while in the disordered one the $J_{i j}$ are chosen as i.i.d. random variables. The partition function in the FK representation is (up to a prefactor)

$$
\mathcal{Z} \sim \sum_{\mathcal{G}} \mathrm{p}^{|\mathcal{G}|}(1-\mathrm{p})^{|\overline{\mathcal{G}}|} q^{|| \mathcal{G}||},
$$

for the pure model, with a straightforward generalization to the random case. Here $\mathcal{G}$ runs over all clusters (i.e. domains connected by the above placed bonds), $|\mathcal{G}|$ is the number of bonds, $|\overline{\mathcal{G}}|$ the not placed bonds, and $\|\mathcal{G}\|$ the number of connected components. The partition sum (1) allows to define the Potts model with non-integer $q \geq 0$. For the pure model it has a continuous phase transition for $0 \leq q \leq 4$, which becomes first order for $q>4$.

Our analytical calculation focuses on weak disorder, where the $J_{i j}=\bar{J}+\delta J_{i j}$ are Gaussian random variables of variance $\beta^{2} \overline{\delta J_{i j}^{2}}=g_{0}$ and $\sqrt{g_{0}} \ll \beta \bar{J}$. Near the critical temperature of the pure model, the continuum limit of the random Potts model can be written [6, 7] as $\mathcal{H}=\mathcal{H}_{\text {pure }}+\int_{\vec{z}} \varepsilon(\vec{z}) \delta J(\vec{z})$ where $\int_{\vec{z}} \equiv \int \mathrm{d}^{2} \vec{z}$ and $\beta \mathcal{H}_{\text {pure is }}$ 
the action of the pure $q$-state Potts model, which at criticality can be identified with its conformal field theory, or the $O(N=\sqrt{q})$ model [12]. We use the Coulomb-gas representation of the latter. The coupling constant $p$ is related to $q$ via $\sqrt{q}=2 \cos (\pi /(2 p))$, so that $p=2$ is Ising and $p=3$ is 3 -state Potts. The second term in $\mathcal{H}$ is the deviation from the pure critical point induced by the disorder, where $\varepsilon(\vec{z})$ is the energy density operator of the pure model. To average over disorder, the $n$-times replicated action is taken:

$$
\ln \overline{e^{-\beta \sum_{a=1}^{n} \mathcal{H}^{a}}}=-\beta \sum_{a=1}^{n} \mathcal{H}_{\text {pure }}^{a}+g_{0} \int_{\vec{z}} \sum_{a, b=1}^{n} \varepsilon^{a}(\vec{z}) \varepsilon^{b}(\vec{z})
$$

where everywhere below we use the shorthand notation $\Phi(z)$ to denote $\Phi(\vec{z})$, where $\vec{z}=(z, \bar{z})$. The diagonal term $\varepsilon^{a}(z)^{2}$ is perturbatively less relevant [6] than $\varepsilon^{a}(z) \varepsilon^{b}(z)$, whose dimension is $4 \Delta_{\varepsilon}$, i.e., four times the dimension of the holomorphic part of the energy primary field $\varepsilon(z) \equiv \Phi_{12}(z)$, with $\Delta_{\varepsilon}=\frac{p+1}{2(2 p-1)}$. For the Ising model, $p=q=2$, and disorder is marginally irrelevant, whereas for the 3 -states Potts model $p=q=3$ it is relevant. Since the Coulomb gas is defined for all $p$, we can perturbatively expand around the Ising model [6]. This expansion is conceptually the same as for the $\phi^{4}$ model, except that Feynman diagrams are evaluated using the unperturbed CFT (with averages denoted $\langle\ldots\rangle_{0}$ ). We keep the perturbed system on its critical manifold, s.t. only the renormalization of the disorder $g_{0}$ is left to consider, with a correction to second order $O\left(g_{0}^{2}\right)$ :

$$
\begin{aligned}
& \sum_{a \neq b} \varepsilon^{a}(z) \varepsilon^{b}(z) \sum_{c \neq d} \varepsilon^{c}\left(z^{\prime}\right) \varepsilon^{d}\left(z^{\prime}\right) \\
& =4(n-2) \sum_{b \neq d} \varepsilon^{b}(z) \varepsilon^{d}(z)\left\langle\varepsilon(z) \varepsilon\left(z^{\prime}\right)\right\rangle_{0}+\cdots .
\end{aligned}
$$

Using the unperturbed average $\left\langle\varepsilon(z) \varepsilon\left(z^{\prime}\right)\right\rangle_{0}=\frac{1}{\left|z-z^{\prime}\right|^{4 \Delta_{\varepsilon}}}$ one obtains the renormalized disorder $g L^{4 \Delta_{\varepsilon}-2}=g_{0}+$ $4 \pi(n-2) g_{0}^{2} \frac{L^{2-4 \Delta_{\varepsilon}}}{2-4 \Delta_{\varepsilon}}, L$ being the infrared cutoff, and the $\beta$-function (for $q>2$ ) [6]:

$$
L \partial_{L} g=\left(2-4 \Delta_{\varepsilon}\right) g+4 \pi(n-2) g^{2}+\cdots
$$

At $n=0, \beta(g)$ has an infrared fixed point at $g^{*}=\frac{1-2 \Delta_{\varepsilon}}{4 \pi}$ which determines the low-energy behavior of the random model. Conformal symmetry is expected to be restored at $g^{*}$. To date, this method has been employed to calculate the scaling dimension of the energy density $\varepsilon$ and of the spin $\sigma$, to two- and three-loop order in [6, 7] and [7] respectively. The multi-scaling properties of spin-spin correlation function has been determined in [9].

Here we focus on geometrical properties, hence on the operator $\Phi_{10}(z)$ which measures [13] the passage of one critical curve at point $\vec{z}$. Indeed, for the pure model, the correlation function $\left\langle\Phi_{10}(z) \Phi_{10}(0)\right\rangle_{0}=|z|^{-4 \Delta_{10}}$ gives the probability that two points lie at the perimeter of the same FK cluster, from which one obtains the fractal dimension of FK domain walls $d_{\mathrm{f}}^{\mathrm{FK} \text {,pure }}=2-2 \Delta_{10}$, i.e.
$d_{\mathrm{f}}^{\mathrm{FK}}=8 / 5$ for $q=3$. Here we compute the corresponding probability for the disordered system. A crucial question is whether $\Phi_{10}(z)$ is still the "curve-detecting" operator in the disordered system. This is true at the "critical dimension" $p=q=2$. Increasing $p$ deforms the operator adiabatically. Since the latter is a physical observable, it is an eigenoperator of the RG. We must check if there is an operator at $p=2$ which (i) has the same dimension as $\Phi_{1,0}$, and (ii) appears in the sub-algebra generated by $\Phi_{1,0}$ and $\Phi_{1,2}$. If such an operator exists, it mixes with $\Phi_{1,0}$, and the curve-detecting operator will be one of the eigenoperators of the RG flow involving $\Phi_{10}$. We checked the absence of such an operator: thus, at least for small $p-2, \Phi_{10}$ is the curve-detecting operator.

We now sketch the calculation of the scaling dimension of $\Phi_{10}$, for details see [16]. There is no contribution to order $g_{0}$, since contracting the disorder operator $\sum_{b \neq c} \varepsilon^{b} \varepsilon^{c}$ with $\Phi_{10}$ in, say, replica $a$, leaves one $\varepsilon$ in replica $b \neq a$, thus is not proportional to $\Phi_{10}^{a}$. At second order, contracting two disorder vertices with $\Phi_{10}^{a}\left(z_{1}\right)$ gives

$$
\Phi_{10}^{a}\left(z_{1}\right) \frac{g_{0}^{2}}{2 !}\left[\sum_{b \neq c} \int_{z_{2}} \varepsilon^{b}\left(z_{2}\right) \varepsilon^{c}\left(z_{2}\right)\right]\left[\sum_{d \neq e} \int_{z_{3}} \varepsilon^{d}\left(z_{3}\right) \varepsilon^{e}\left(z_{3}\right)\right]
$$

and projecting onto $\Phi_{10}^{a}\left(z_{1}\right)$. Contracting using $\left\langle\varepsilon^{c}\left(z_{2}\right) \varepsilon^{e}\left(z_{3}\right)\right\rangle_{0}=\delta^{c e}\left|z_{2}-z_{3}\right|^{-4 \Delta_{12}}$ to eliminate replicas not equal to $a$ we obtain $\Phi_{10}^{a}\left(z_{1}\right) \varepsilon^{b=a}\left(z_{2}\right) \varepsilon^{d=a}\left(z_{3}\right)$, which, projected onto $\Phi_{10}^{a}\left(z_{1}\right)$ yields

$$
\left\{\begin{aligned}
z_{1} & \frac{g_{0}^{2}}{2 !} 4(n-1) \int_{z_{2}, z_{3}}\left\langle\varepsilon\left(z_{2}\right) \varepsilon\left(z_{3}\right)\right\rangle_{0} \\
& \times\left(\Phi_{10}\left(z_{1}\right) \varepsilon\left(z_{2}\right) \varepsilon\left(z_{3}\right) \mid \Phi_{10}\left(z_{1}\right)\right),
\end{aligned}\right.
$$

where the OPE coefficient $\left(\Phi_{10}\left(z_{1}\right) \varepsilon\left(z_{2}\right) \varepsilon\left(z_{3}\right) \mid \Phi_{10}\left(z_{1}\right)\right):=$ $\lim _{R \rightarrow \infty} \frac{\left\langle\Phi_{10}\left(z_{1}\right) \varepsilon\left(z_{2}\right) \varepsilon\left(z_{3}\right) \Phi_{10}(R)\right\rangle_{0}}{\left\langle\Phi_{10}\left(z_{1}\right) \Phi_{10}(R)\right\rangle_{0}}$. This and the integral (4) are computed using Coulomb gas techniques [11. One $2 \mathrm{~d}$ integration, over one angle and one scale, is easy, and gives a pole in $1 /(p-2)$. One $2 \mathrm{~d}$ integral over say $z_{2}$ is left, but we also need a screening charge $V_{+}$to get the 4-point function in (4). We evaluated this integral in the marginal dimension, i.e. for $p=2$ (Ising) by analytical techniques [7, and numerically [16]. The result is

$$
\begin{aligned}
& \int_{z_{2}, z_{3}}\left(\Phi_{10}\left(z_{1}\right) \varepsilon\left(z_{2}\right) \varepsilon\left(z_{3}\right) \mid \Phi_{10}\left(z_{1}\right)\right)\left\langle\varepsilon\left(z_{2}\right) \varepsilon\left(z_{3}\right)\right\rangle \\
& =-7.0710 L^{4-8 \Delta_{12}}\left(1-2 \Delta_{12}\right)^{-1}
\end{aligned}
$$

Inserting the fixed-point value $g^{*}$ from above gives $\operatorname{dim}_{L}\left(\Phi_{10}\right)=-2 \Delta_{10}+\frac{\left(1-2 \Delta_{\varepsilon}\right)^{2}}{2 \pi^{2}} \times 7.071 \stackrel{p=3}{=}-\frac{2}{5}+0.01433$. This leads to the fractal dimension of FK domain walls:

$$
d_{\mathrm{f}}^{\mathrm{FK}}=2+\operatorname{dim}_{L}\left(\Phi_{10}\right)=1.61433 .
$$

Let us note a few additional peculiar features which come out of the calculation [16]. The 4-point function

$$
G(u):=\lim _{\left|z_{4}\right| \rightarrow \infty}\left|z_{4}\right|^{4 \Delta_{10}}\left\langle\Phi_{10}(0) \varepsilon(1) \varepsilon(u) \Phi_{10}\left(z_{4}\right)\right\rangle
$$




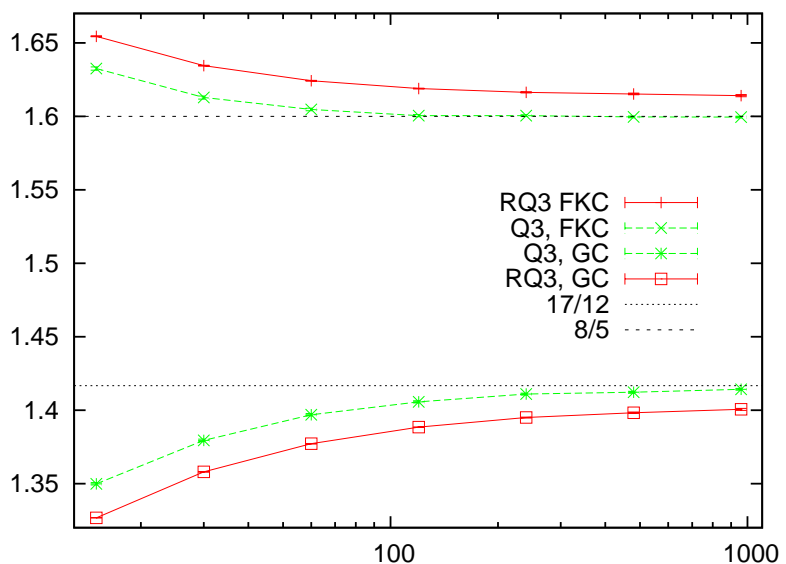

FIG. 1: Fractal dimension of FK and spin clusters, both for the pure (green) and disordered (red) system, using the Wolff algorithm.

at $p=2$, i.e., for the Ising model is

$$
\begin{aligned}
& \left.G(u)\right|_{p=2}=\left.\left.\frac{\Gamma\left(\frac{1}{3}\right)^{6}}{27 \pi^{2}} \frac{|u|^{\frac{2}{3}}}{|1-u|^{2}}\right|_{2} F_{1}\left(-\frac{1}{3}, \frac{2}{3} ; 2 ; u\right)\right|^{2} \\
& +\frac{\Gamma\left(\frac{1}{3}\right)^{8}}{54 \sqrt{3} \pi^{3}} \frac{|u|^{\frac{2}{3}}}{|1-u|^{2}}\left[{ }_{2} F_{1}\left(-\frac{1}{3}, \frac{2}{3} ; 2 ; u\right) G_{2,2}^{2,0}\left(\bar{u} \mid \begin{array}{c}
\frac{1}{3}, \frac{4}{3} \\
-1,0
\end{array}\right)+c . c .\right]
\end{aligned}
$$

$G$ is the Meijer $G$-function, which has a logarithmic divergence at $u=0$,

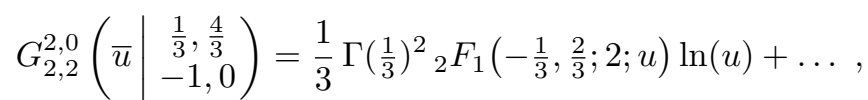

dropping regular terms. The structure of the result and the logarithmic divergence remain valid for larger values of $p$, with the parameters replaced by rational functions of $p$. This behavior is consistent with the appearance of operators of canonical dimensions 1 and 0 (different from the identity) in the OPE of $\varepsilon$ with $\Phi_{1,0}$ as discussed in a similar case in [17. Logarithms are known to appear for operators on the boundary of the Kac table [18] and in disordered systems 24].

Even more surprisingly, we attempted to perform the same calculation for the fractal dimension of spin interfaces, using the operator $\Phi_{01}$ as curve detector. There the equivalent of (7) does not exist: At least we were not able to construct a 4-point function, which satisfies the differential equation induced by the 0 -vector condition associated to $\varepsilon=\Phi_{12}$ at level 2, which is unique-valued, and reproduces the correct OPE in the limit of $u \rightarrow 1$.

We now discuss our numerical results. For the Monte Carlo simulations we use the Wolff cluster algorithm [10. It consists in randomly choosing a spin, and then joining with probability $\mathrm{p}$ nearest-neighbor spins to belong to the same cluster. This procedure is repeated until no nearest neighbor can be joined anymore. $p$ itself is a quenched random variable on the edges of the lattice, taken from the symmetric bimodal distribution $\left\{\mathrm{p}_{1}, \mathrm{p}_{2}\right\}=\left\{1-\exp \left(-\beta_{c} J_{1}\right), 1-\exp \left(-\beta_{c} J_{2}\right)\right\}$. The choice $\mathrm{p}_{1} \mathrm{p}_{2}=q$ ensures that the system is at its critical point 14. We use $J_{1}=J_{2}$ for the pure, and $J_{1} / J_{2}=10$ for the random-bond disordered system. Once the cluster is constructed, we assign to it with equal probability one of the $q$ colors, as long as this is consistent with the boundary conditions (BC) discussed below. Wolff has shown [10] that this algorithm produces the correct statistical weight of the Potts model, is ergodic, and that the critical slowing down is reduced compared to ordinary Monte Carlo algorithms. In addition, it allows to track both spin and FK clusters. In the simulations, we imposed boundary conditions which create a domain wall which span the lattice as was already done by Gamsa and Cardy [15. We considered various types of conformally invariant boundary conditions: "fluctuating" $(a / \bar{a})$, "fixed" $(a / b)$, and "free" ( $a /$ free $)$, all of them giving the same result in the large-size limit.

We measured the fractal dimension from the average length $l$ of the domain wall as a function of the linear size $L$ of the lattice $\overline{\langle l\rangle} \simeq L^{d_{\mathrm{f}}}$, where $\langle\cdots\rangle$ denotes the thermal average and $\cdots$ the disorder average $[25]$. The results presented here are obtained with a thermal average over $\simeq 10^{6} \times \tau$ for the pure system and a disorder average over $\simeq 10^{5}$ configurations for the disordered system. $\tau$ is the autocorrelation time which was first determined for each size, see [16] for details. Our simulations show that for the pure system, all these domain walls have asymptotically the same fractal dimension, with the exception of the common domain wall for fixed BC, which has dimension one. In Fig. 1 we plot the effective fractal dimension versus $L$. As $L \rightarrow \infty$ the fractal dimensions of the pure system converge to the values predicted by conformal field theory, $d_{\mathrm{f}}^{\text {spin }}=\frac{17}{12}$, and $d_{\mathrm{f}}^{\mathrm{FK}}=\frac{8}{5}$, corroborating partial results by Gamsa and Cardy [15]. Our estimate from all BC, extrapolated to an infinite system gives $d_{\mathrm{f}}^{\text {spin }}=1.416 \pm 0.002$, and $d_{\mathrm{f}}^{\mathrm{FK}}=1.599 \pm 0.002$. For the disordered system we find

$$
d_{\mathrm{f}}^{\text {spin }}=1.401 \pm 0.003, \quad d_{\mathrm{f}}^{\mathrm{FK}}=1.614 \pm 0.003
$$

This is in excellent agreement with our analytical result (6). We have also checked the SLE duality relation $\kappa \kappa^{\prime}=16$. Using $d_{\kappa}=1+\frac{\kappa}{8}$, we find for the pure system $\kappa^{\text {spin }}=3.328 \pm 0.016, \kappa^{\mathrm{FK}}=4.792 \pm 0.016$, and $\kappa^{\mathrm{spin}} \kappa^{\mathrm{FK}}=15.95 \pm 0.13$. For the disordered system, we find $\kappa^{\text {spin }}=3.208 \pm 0.024, \kappa^{\mathrm{FK}}=4.912 \pm 0.024$, and $\kappa^{\mathrm{spin}} \kappa^{\mathrm{FK}}=15.76 \pm 0.20$.

In the transfer-matrix approach, we studied the FK clusters in the equivalent loop formulation [19]. The loops are defined on the medial lattice as the external and internal hulls of the FK clusters. The random bonds were again drawn from a bimodal distribution, with an equal number of strong and weak bonds 21. The strength of the disorder is conveniently characterized by the parameter $s$ [20] defined by $J_{1} / J_{2}=\ln (1+s \sqrt{q}) / \ln (1+\sqrt{q} / s)$, 


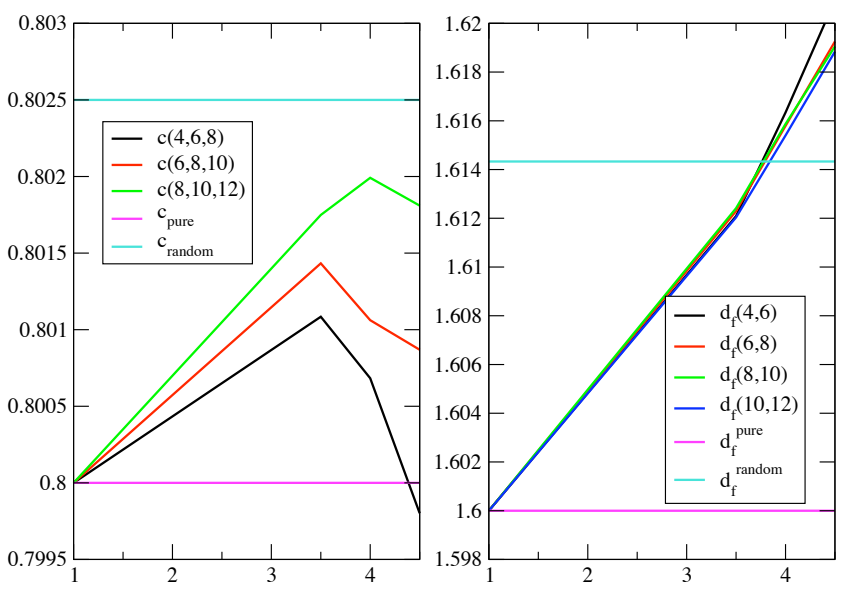

FIG. 2: Effective central charges $c(L)$ and fractal dimensions $d_{\mathrm{f}}^{\mathrm{FK}}(L)$ versus disorder strenght $s$, for $s=3.5,4.0,4.5$. The linear interpolation is only a guide to the eye. Each curve has been normalized so that finite-size effects are absent for $s=1$ (no disorder). Horizontal lines give corresponding exact (resp. perturbative) values for the pure (resp. disordered) system.

with $q=3$. For a given fixed realization of the random bonds on long cylinders of length $M=10^{5}$ and circumference $L=4,6, \ldots, 12$ lattice spacings (for the medial lattice) the corresponding free energy $f_{j}(L)$, normalized per lattice site, was computed from the leading Lyapunov exponent of the corresponding product of random transfer matrices. The transfer direction was taken axial with respect to the medial lattice (hence diagonal with respect to the original square lattice supporting the Potts spins) [19. Three different topological sectors were considered, corresponding to enforcing $j=0,2,4$ loop segments to propagate along the length direction of the cylinder. The fluctuations of these free energies were studied by averaging over at least $M^{\prime}=10^{5}$ independent cylinders.

Conformal field theory predicts [22] that $\overline{f_{0}(L)}=$ $\overline{f_{0}(\infty)}-\frac{\pi c}{6 L^{2}}+\frac{A}{L^{4}}+\cdots$, where $c$ is the effective central charge and $A$ a non-universal constant. Applying this to three consecutive $L$ gives estimates $c(L-4, L-2, L)$ shown in the left panel of Fig. 2. The fixed-point value $s^{*}$ of the disorder strength corresponds to the locus of the maximum of $c$, and is estimated as $s^{*}=4.0 \pm 0.3$ (using also data not shown here), improving on the value $s^{*}=3.5 \pm 0.5$ reported earlier [20]. The effective central charge of the disordered model is estimated as $c\left(s^{*}\right)=0.8024 \pm 0.0003$, in excellent agreement with the three-loop perturbative result [7] $c \simeq 0.8025$.

Correlation functions $G_{j}(M)$ are defined as the probability of having $j$ loop segments propagate over a distance $M$ along the cylinder axis without joining up. They are related to the free energy gaps through $\Delta f_{j}(L) \equiv$ $f_{j}(L)-f_{0}(L)=\frac{-1}{M L} \ln G_{j}(M)$. Their disorder-averaged $n$ 'th moment can be extracted from the cumulant expansion 8$] \ln \overline{\left(G_{j}\right)^{n}}=n \overline{\ln G_{j}}+\frac{1}{2} n^{2} \overline{\left(\ln G_{j}-\overline{\ln G_{j}}\right)^{2}}+\cdots$, where the quantities on the r.h.s. are self-averaging. Only the first two cumulants contribute significantly. CFT predicts [23] that $\frac{-1}{M L} \ln \overline{\left(G_{j}\right)^{n}}=\frac{2 \pi x_{j}}{L^{2}}+\frac{B}{L^{4}}+\cdots$, where the $n$-dependent conformal weights $x_{j}$ are related to the desired (multi)fractal dimensions via $d_{j}=2-x_{j}$. For $n=1$, we have $d_{2}=d_{\mathrm{f}}^{\mathrm{FK}}$ defined above; $d_{4}$ gives the dimension of "red bonds" (whose removal disconnects a cluster). As seen from the right panel of Fig. 2, the effective values of $d_{j}$ depend strongly on $s$, so accuracy for $s^{*}$ is important 20. Using $s^{*}=4.0 \pm 0.3$ we estimate $d_{2}=1.615 \pm 0.002$, in excellent agreement with (6) and (8).

To conclude, our analytical and numerical results for the fractal dimension of FK domain walls agree well. Fractal dimensions of spin interfaces have been determined from numerics and seem in agreement with the duality relation suggested by SLE. Pending questions under investigation are possible multiscaling, the fractal dimensions of spin interfaces and SLE type observables.

We thank M. Bauer, D. Bernard, Vl. Dotsenko, A. Ludwig, T. Quella and P. Wiegmann for valuable discussions. Supported by ANR (05-BLAN-0099-01).

[1] O. Schramm, Israel J. Math. 118, 221, (2000).

[2] Reviews: M. Bauer and D. Bernard, Phys. Rep. 432 (2006) 115. J. Cardy, Ann. Phys. 318 (2005) 81.

[3] R. Santachiara, Nucl. Phys. B 793 (2008) 396; M. Picco and R. Santachiara, Phys. Rev. Lett. 100 (2008) 015704.

[4] D. Bernard et al. Nature Physics 2124 (2006).

[5] D. Bernard, et al., Phys. Rev. B 76 (2007) 020403 (R) .

[6] A.W.W. Ludwig, Nucl. Phys. B 285 (1987) 97; A.W.W. Ludwig and J. Cardy, ibid., 687.

[7] Vl.S. Dotsenko, et al., Nucl. Phys. B 455 (1995) 701.

[8] M. Picco, Phys. Rev. Lett. 792998 (1997); J. Cardy and J.L. Jacobsen, Phys. Rev. Lett. 79, 4063 (1997); J.L. Jacobsen and J. Cardy, Nucl. Phys. B 515, 701 (1998).

[9] A.W.W. Ludwig, Nucl. Phys. B 330 (1990) 639; Vik.S. Dotsenko, et al., Nucl. Phys. B 520 (1998) 633; M.A. Lewis, Europhys. Lett. 43 (1998) 189.

[10] U. Wolff, Phys. Rev. Lett. 62 (1989) 361.

[11] Vl.S. Dotsenko, cel.archives-ouvertes.fr/cel-00092929

[12] B. Nienhuis, J. Stat. Phys. 34 (1984) 731.

[13] I. Rushkin, et al., J. Phys. A 40 (2007) 2165; H.W.J. Blöte, et al., Phys. Rev. Lett. 68 (1992) 3440.

[14] W. Kinzel and E. Domany, Phys. Rev. B 23 (1981) 3421.

[15] A. Gamsa and J. Cardy, J. Stat. Mech. (2007) P08020.

[16] Present authors, in preparation.

[17] V. Gurarie, Nucl. Phys. B410 (1993) 535.

[18] P.A. Pearce, et al., J. Stat. Mech. 0611 (2006) P017.

[19] Vl.S. Dotsenko, et al., Nucl. Phys. B 546, 505 (1999).

[20] J.L. Jacobsen, Phys. Rev. E 61, R6060 (2000).

[21] J.L. Jacobsen and M. Picco, Phys. Rev. E 61, R13 (2000).

[22] H.W.J. Blöte, et al. Phys. Rev. Lett. 56, 742 (1986); I. Affleck, Phys. Rev. Lett. 56, 746 (1986).

[23] J. Cardy, J. Phys. A 17, L385 (1984).

[24] J. Cardy, cond-mat/9911024; S. Caux, hep-th/9511134.

[25] Measurements of the moments $\overline{\langle l\rangle^{m}}$ for $m=1,2, \ldots$ are consistent with an unambiguous definition of $d_{\mathrm{f}}$. 